\title{
The prevalence and risk factors for urinary tract infection in elderly patients presenting with proximal femoral fractures: a prospective observational study
}

\author{
Pretorius $\mathrm{C}^{1}$, Immelman $\mathrm{RJ}^{2}$, Ismail $\mathrm{F}^{3}$, Ngcelwane $\mathrm{MV}^{4}$, Motsitsi $\mathrm{NS}^{5}$ \\ MBChB(Wits), MMed(Orth)(Pret) (5th year), FC(Orth)(SA); Orthopaedic Surgeon, Steve Biko Academic Hospital, Pretoria \\ 2 MBChB(Pret), MMed(Orth)(Pret), FC(Orth)(SA); Orthopaedic Surgeon, Kalafong Hospital, Pretoria \\ 3 BSc, MBChB(Pret), DTM\&H(Wits), MMed(Medical Microbiology)(Pret); Medical Microbiologist, National Institute of Communicable Diseases/National \\ Health Laboratory Services, Department of Medical Microbiology, University of Pretoria \\ 4 MBChB(Natal), FCS(Orth)(SA), MSc(Orth)(London); Professor \& Head of Department of Orthopaedic Surgery, Steve Biko Academic Hospital, \\ Pretoria \\ 5 MBChB, MMed(Orth)(Medunsa), FCS(ASEA); Professor \& Head of Department of Orthopaedic Surgery, Kalafong Hospital MBChB(Natal), FCS(Orth) \\ (SA), MSc(Orth)(London); Professor \& Head of Department of Orthopaedic Surgery, Steve Biko Academic Hospital, Pretoria, Pretoria
}

Corresponding author: $\operatorname{Dr} C$ Pretorius, Department of Orthopaedic Surgery, Steve Biko Academic Hospital, University of Pretoria, 11436+

Tom Jenkins Drive, Rietondale, Pretoria; cell: 072994 1823; email: drcpretorius@gmail.com

\begin{abstract}
Background: Proximal femoral insufficiency fractures are increasingly common in the elderly population. Urinary tract infection (UTI) is the most common associated complication in this group of patients with these injuries and may add to an already severe morbidity and mortality. The incidence of patients presenting with an established UTI from home is unknown as most research focuses on UTIs that occur after hospital admission. The aim of the study was to determine the prevalence of UTIs on admission and to compare urine culture results and patient profiles of these patients.

Methods: Forty patients presenting with a proximal femoral insufficiency fracture were identified on admission to casualty. A urine specimen was taken according to a standard protocol and sent for microscopy, culture and sensitivity (MCS). The organism and sensitivity profiles were determined from all positive MCS samples. Risk factors and patient demographics were identified using a questionnaire.

Results: Twenty of the 40 patients (50\%) had a positive culture from the urine MCS. The most common organism was Escherichia coli $(55 \%)[\mathrm{Cl}=33.2 ; 76.8 \%]$ and the most common antibiotic sensitivity was to amoxicillin/clavulanic acid. Most of the organisms identified were resistant to ampicillin/amoxicillin and trimethoprim/sulfamethoxazole. Significant risk factors associated with an increased prevalence were female sex and the age of the patient.

Conclusion: The prevalence of UTI in elderly patients with proximal femoral fractures is underestimated. Higher risk patients for presenting with a UTI from home are the elderly female patients above 80 years of age. Screening for, diagnosing and treating UTIs earlier has clear benefits for this specific group of patients to potentially decrease overall morbidity and mortality.
\end{abstract}

Level of evidence: Level 4

Key words: proximal femoral insufficiency fractures, urinary tract infection, urine microscopy culture and sensitivity, risk factors, prevalence

Citation: Pretorius C, Immelman RJ, Ismail F, Ngcelwane MV, Motsitsi NS. The prevalence and risk factors for urinary tract infection in elderly patients presenting with proximal femoral fractures: a prospective observational study. SA Orthop J 2018;17(4):27-32. http://dx.doi.org/10.17159/2309-8309/2018/v17n4a3

Editor: Prof S Maqungo, University of Cape Town

Received: March 2018

Accepted: August 2018

Published: November 2018

Copyright: ( 2018 Pretorius C. This is an open-access article distributed under the terms of the Creative Commons Attribution Licence, which permits unrestricted use, distribution and reproduction in any medium, provided the original author and source are credited.

Funding: No funding was received for this research.

Conflict of interest: All authors of this article declare that there was no conflict of interest with respect to this study. 


\section{Introduction}

Patients with proximal femoral fractures are known to suffer from many complications ranging from delirium, pneumonia, venous thromboembolism and bed sores. However, none of these are as common as urinary tract infection (UTI). ${ }^{1}$ With our elderly population growing, there is an increase in the incidence of proximal femoral insufficiency fractures. These fractures pose a major problem to health care workers and place great constraints on our health care system. Due to many physiological constraints on an already fragile system, underlying minor conditions like a UTI can have a marked effect on patient morbidity and mortality.

Many patients develop a UTI during the hospital stay with an estimated incidence of $>25 \%$ of patients affected. However, the prevalence of UTI already present before or at admission from home is unknown. ${ }^{1-3}$ We could only find a few studies that looked specifically at the prevalence of UTI at presentation. The prevalence of UTI on arrival at hospital and admission is reported as $16 \%$ with results being defined as a positive urine culture. This study by Dolk et al. was done in 1989 and since then our population and microbiological profile has changed significantly. ${ }^{2}$

In this prospective study the aim was to determine the prevalence of UTI in all elderly patients presenting with proximal femoral insufficiency fractures from home by collecting urine samples on presentation in casualty, and thus establishing the true incidence in our specific population. We also aimed to determine the relevant risk factors for presenting with a UTI, as well as to identify the causative organisms and their sensitivity profile.

\section{Materials and methods}

This prospective observational study was conducted in the Department of Orthopaedic Surgery at Steve Biko Academic Hospital in Pretoria between November 2015 and July 2017. All patients aged 65 years and older presenting with a proximal femoral insufficiency fracture were included. The term 'proximal femoral insufficiency fractures' included both neck and intertrochanteric femur fractures. These patients were screened for UTIs and their specific patient demographics recorded using a standard questionnaire. Exclusion criteria used were for patients presenting with a high energy traumatic fracture as well as those that had a urinary catheter insertion pre-admission. If the duration between the injury and admission was more than seven days or if the patients had received any antibiotics within the last three months, they were also excluded.

All patients with proximal femur fractures are routinely catheterised as is the normal protocol in our unit with a urine sample sent for urine microscopy, culture and sensitivity (U-MCS). The collection and testing protocol was devised in conjunction with the Department of Medical Microbiology at the University of

Table I: Patient demographics and risk factors for UTI

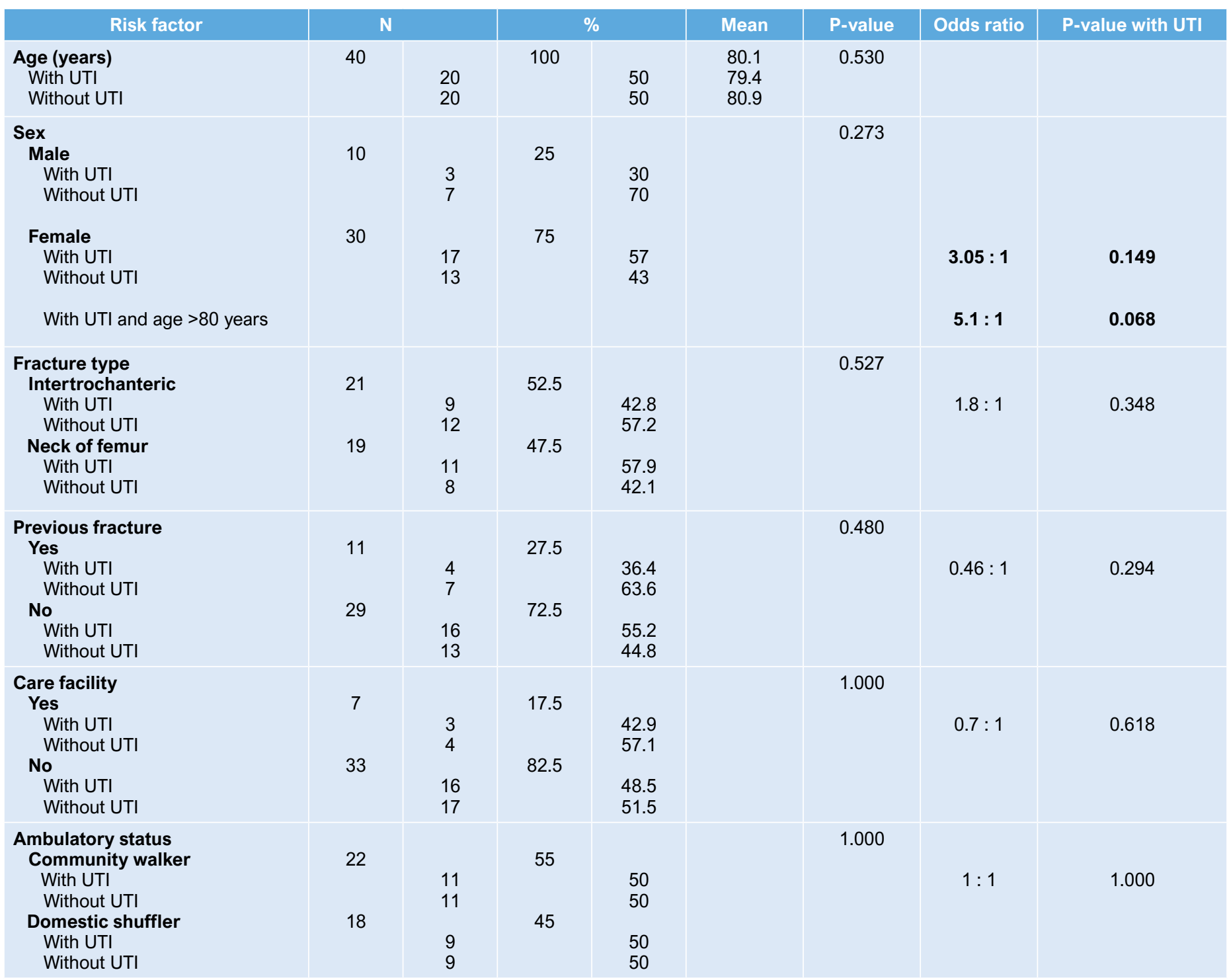


Pretoria. The collection of urine samples from the patients that met the inclusion criteria was done using a standard sterile protocol each time. Patients had a urethral catheter placed under sterile conditions by a qualified medical doctor. A clean-catch urine specimen was collected and taken to the National Health Laboratory Service (NHLS) immediately by a member of the orthopaedic oncall team for urine culture so that it could be processed within two hours. Urine samples were processed and tested in accordance to the NHLS standard operating procedures. The urine samples were processed in a semi-quantitative manner using calibrated loops $(1 \mu \mathrm{l})$ and plated onto Uriselect ${ }^{\mathrm{TM}} 4$ (Bio-Rad, California, USA), a non-selective chromogenic agar medium. This medium is used for the isolation, differentiation and enumeration of common urinary tract pathogens. Identification and susceptibility of bacteria was confirmed by the VITEK 2 automated identification system (bioMerieux, Marcy l'Etoile, France). The U-MCS results were all verified and supervised by a qualified medical microbiologist.

A UTI was deemed positive when the urine culture had $\geq 10^{5}$ colony-forming units (CFU)/ml with no more than two species of microorganisms cultured. ${ }^{4}$ This is in accordance with the Centre for Disease Control (CDC) and the NHLS standard operating procedure for urines samples. Mixed results were reported when more than two species of bacteria were isolated; these were considered as a possible contamination and excluded. Additional data collected included the identified organisms and the sensitivity of the organism cultured.

Patient demographics and risk factors that were collected included age, sex and type of fracture. Other risk factors that were looked at were whether the patient was residing in an old-age home or a care facility. Ambulatory status as well as history of previous fractures was also included. These risk factors and demographics were captured using a questionnaire with 'yes' or 'no' answer questions, together with an informed consent form. All patients in the study gave informed consent. Patients who chose not to participate in the study received the same standard treatment and were not disadvantaged. Statistical analysis of data was done via cross-tabulation using the Fisher exact and chi-squared tests.

\section{Results}

In this study period there were 40 patients with proximal femoral insufficiency fractures that met the inclusion criteria. Of the 40 patients, 22 had a positive urine culture. Two patients were excluded because they had a mixed growth shown on their U-MCS. We were left with 20 patients with a positive urine sample (U-MCS).

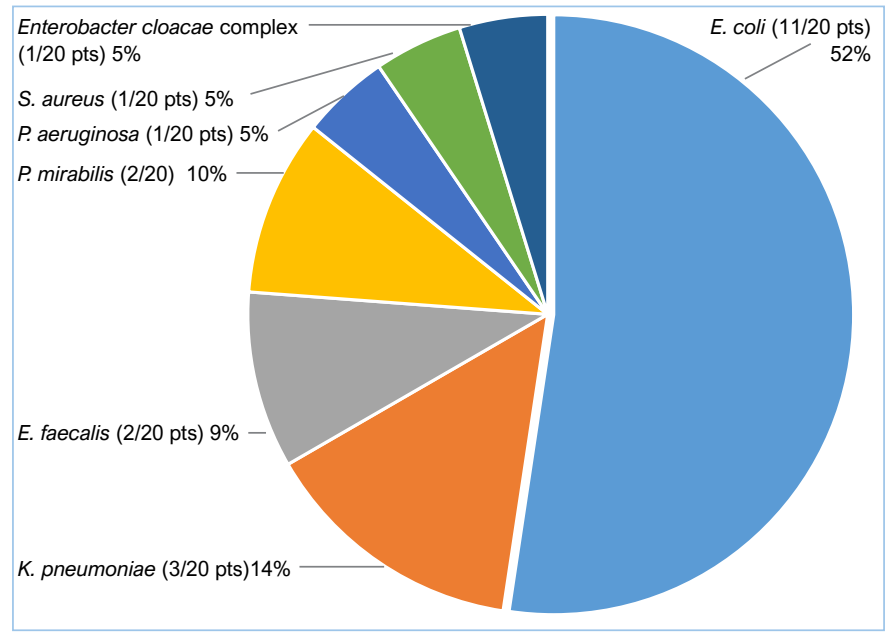

Figure 1. Distribution of organisms identified on U-MCS of 20 patients with positive cultures

Our study thus shows a prevalence rate of $50 \%$ for all patients presenting with UTI pre-admission, and from home with a $57 \%$ and $30 \%$ prevalence for females and males, respectively. The mean age of all participants was 80.1 years with $30(75 \%)$ being female and ten $(25 \%)$ male. A summary of the demographics and risk factors is shown in Table $I$.

The most common organism identified was Escherichia coli, cultured in 11 of the 20 patients with positive cultures (55\%) $[\mathrm{Cl}=33.2 ; 76.8 \%]$. Other organisms cultured included Klebsiella pneumoniae $(3 / 20 ; 15 \%)$, Enterococcus faecalis $(2 / 20 ; 10 \%)$, Proteus mirabilis $(2 / 20 ; 10 \%)$, Pseudomonas aeruginosa (1/20; $5 \%)$, Staphylococcus aureus (1/20; $5 \%$ ) and Enterobacter cloacae complex $(1 / 20 ; 5 \%)$. Two samples that cultured a mixed result were excluded. The distribution of organisms cultured is shown in Figure 1.

The sensitivity profile to commonly used antibiotics of the 11 Escherichia coli organisms that were cultured is shown in Table II as well as that of the other organisms identified in Table III. All 11 Escherichia coli were sensitive to amoxicillin/clavulanic acid.

\section{Discussion}

Patients in the geriatric population have vastly improved their life expectancy and are living longer, resulting in an ever-increasing number of patients in this group requiring treatment. In this

Table II: Antibiotic sensitivity profile of the Escherichia coli organism cultured in 11 of the patients with positive urine cultures

\begin{tabular}{|c|c|c|c|c|c|c|}
\hline $\begin{array}{l}\text { E.coli } \\
11 \text { pts }\end{array}$ & $\begin{array}{l}\text { Amoxicillin/ } \\
\text { ampicillin }\end{array}$ & $\begin{array}{c}\text { Trimethoprim/ } \\
\text { sulfamethoxazole }\end{array}$ & Ciprofloxacin & Nitrofurantoin & Co-amoxiclav & Cephalosporins \\
\hline 1 & $\mathrm{R}$ & $\mathrm{R}$ & $\mathrm{R}$ & NS & $\checkmark$ & $\checkmark$ \\
\hline 2 & $\checkmark$ & $\checkmark$ & $\mathrm{R}$ & $\checkmark$ & $\checkmark$ & $\checkmark$ \\
\hline 3 & $\mathrm{R}$ & $\mathrm{R}$ & $\checkmark$ & NS & $\checkmark$ & $\checkmark$ \\
\hline 4 & $\mathrm{R}$ & NS & $\checkmark$ & $\checkmark$ & $\checkmark$ & $\checkmark$ \\
\hline 5 & $\mathrm{R}$ & NS & $\checkmark$ & $\checkmark$ & $\checkmark$ & $\checkmark$ \\
\hline 6 & $\mathrm{R}$ & $\mathrm{R}$ & $\checkmark$ & $\checkmark$ & $\checkmark$ & NS \\
\hline 7 & $\mathrm{R}$ & $\mathrm{R}$ & $\mathrm{R}$ & $\checkmark$ & $\checkmark$ & NS \\
\hline 8 & $\mathrm{R}$ & $\mathrm{R}$ & $\checkmark$ & NS & $\checkmark$ & $\checkmark$ \\
\hline 9 & $\mathrm{R}$ & $\mathrm{R}$ & $\checkmark$ & $\checkmark$ & $\checkmark$ & $\checkmark$ \\
\hline 10 & $\checkmark$ & $\checkmark$ & $\checkmark$ & $\checkmark$ & $\checkmark$ & $\checkmark$ \\
\hline 11 & $\mathrm{R}$ & $\mathrm{R}$ & $\checkmark$ & $\mathrm{R}$ & $\checkmark$ & NS \\
\hline
\end{tabular}

Cephalosporins $=$ cefazolin (1st generation), cefuroxime (2nd generation), cefotaxime (3rd generation) 
Table III: Antibiotic sensitivity profile of the other organisms cultured

\begin{tabular}{|c|c|c|}
\hline Organism & Sensitivity & Resistance \\
\hline Klebsiella pneumoniae & $\begin{array}{l}\text { Amoxicillin/clavulanic acid, trimethoprim/ } \\
\text { sulfamethoxazole, ciprofloxacin }\end{array}$ & $\begin{array}{l}\text { Amoxicillin/ampicillin, nitrofurantoin } \\
\text { (one organism was resistant to trimethoprim/ } \\
\text { sulfamethoxazole and } 2 \text { nd generation cephalosporins) }\end{array}$ \\
\hline Enterococcus faecalis & Vancomycin, gentamicin & Amoxicillin/ampicillin \\
\hline Proteus mirabilis & $\begin{array}{l}\text { Amoxicillin/clavulanic acid, ciprofloxacin and all } \\
\text { cephalosporins }\end{array}$ & Amoxicillin/ampicillin \\
\hline Pseudomonas aeruginosa & Cefepime, ceftazidime & Amikacin \\
\hline Staphylococcus aureus & Cloxacillin, trimethoprim/sulfamethoxazole & Amoxicillin/ampicillin \\
\hline Enterobacter cloacae complex & Cefepime, ertapenem & $\begin{array}{l}\text { 1st, 2nd and 3rd generation cephalosporins, } \\
\text { ciprofloxacin, gentamicin, amoxicillin/clavulanic acid }\end{array}$ \\
\hline
\end{tabular}

specific patient demographic, the incidence of proximal femoral insufficiency fractures is higher than ever and still on the rise..$^{5,6}$ With the prevalence of these fractures expected to double between 1990 and 2040 and with no real advances in outcomes, more innovative treatment plans have to be considered. ${ }^{5}$ Globally the mean mortality rates of these patients are currently $30 \%$ at six months post trauma, with a massive $50 \%$ of patients losing their independence post injury. ${ }^{6}$

This problem has led to the implementation of protocols in the management of patients with proximal femoral insufficiency fractures with specific emphasis on prevention, early diagnosis and goal-directed treatment of complications, as well as the condition itself. The shift has gone from single care to care involving an entire orthogeriatric team that includes multiple health care professionals to treat and prevent specific complications. ${ }^{5}$

Many of these elderly patients have several comorbidities and their functional reserve is not that of younger patients. As a result, these patients often suffer from many complications after a proximal femur fracture as well as the surgery that follows. These complications rather than the fracture itself have been shown to play a major role in functional decline, increased mortality and prolonged hospital stay. ${ }^{7}$

The most common complication in patients with proximal femoral insufficiency fractures is UTI. ${ }^{1}$ Many patients develop a UTI during the hospital stay with an estimated incidence of $>25 \%$ of patients affected. ${ }^{1-3}$ From the study by Dolk et al. of 282 patients with hip fractures, the prevalence of UTI already present before or at admission from home was $16 \% .^{2}$ The relevance of a high rate of UTI pre-injury and on presentation is that with earlier diagnosis and management of these UTIs one could potentially minimise patient morbidity and mortality.

Results from our study show a prevalence of $50 \%$ with 20 of the 40 patients included presenting with a positive urine culture. The prevalence of pre-existing UTIs from home is thus higher than initially expected in our group of patients. Our study specifically looked at those patients who presented with an established UTI from home as opposed to those who develop a UTI during the stay in hospital. This high prevalence places our specific population at increased risk of morbidity and mortality.

Elderly patients often suffer from many age-related chronic comorbidities that may require a prolonged work up or resuscitation pre-operatively. One of these common comorbidities is UTI. This may lead to an increase in the time from injury until time of surgery. McGuire et al. in a large multicentre study consisting of 18209 patients treated found that a delay in surgery beyond 48 hours showed a $17 \%$ increase in the 30 -day mortality. ${ }^{8}$ These figures have been reciprocated multiple times, all showing that a delay in surgery for more than 48-72 hours is linked to increased morbidity and mortality. International guidelines thus suggest that surgery should take place within $24-48$ hours from injury.8.9 With this time frame in mind, urine for MCS taken on admission is unlikely to show a result at the time of surgery or even by the first day post-operatively. By knowing the true prevalence of UTI at first presentation and which patients are at risk of presenting with a UTI enables one to act and intervene earlier. This improves empirical treatment decisions in clinically suspicious cases without confirmed infections where the organisms and sensitivities are not yet available.

Prosthetic joint infection (PJI) remains a great challenge facing orthopaedic surgeons worldwide. Most research is focused on elective arthroplasty and shows a risk of about $1 \%$ for PJI for patients treated electively for osteoarthritis. Elderly patients (>65 years) with proximal femoral fractures, however, represent a distinct group at higher risk of PJI and should not be viewed the same as patients undergoing elective hip arthroplasty. This incidence increases exponentially in cases of traumatic joint replacements where PJI rates are as high as $9 \%$. The mortality and morbidity rates are substantially higher, with mortality going up from $21 \%$ in the non-infected group to $47 \%$ in the group with PJI. ${ }^{10,11}$ Literature has shown that patients who experience any complications during their hospitalisation for proximal femoral insufficiency fractures have a threefold increase in one-year mortality when compared to patients who have no complications. ${ }^{7}$

UTIs have not been linked to be directly causative of PJI, but have been shown, together with increased waiting time to surgery, to be a statistically significant risk factor for development of PJI. ${ }^{10}$ Yassa et al. did a retrospective analysis in the UK of 367 patients with a high prevalence rate of pre-operative UTI following proximal femur fracture and also found a strong relationship between preoperative UTI and the development of post-operative surgical site infection. ${ }^{12}$ The organisms identified from a PJI are different in many cases from the organism causing the UTI, thus further emphasising that it is not usually directly causative but rather a significant risk factor.

UTIs are also considered an important risk factor for delirium and contribute to prolonged hospital stay for another two to three days on average. Early treatment of UTI decreased the risk of delirium, thus further decreasing hospital stay and improving patient morbidity. ${ }^{12-14}$

In the South African health care system there is great emphasis on saving money and decreasing the cost of health care. Research has substantiated this clear financial implication and shows that almost $20 \%$ more money is being spent per patient with complications, when compared to uncomplicated cases. ${ }^{3}$ UTIs are shown to predispose patients to a longer hospital stay (up to five days) and this may further increase the development of nosocomial complications contributing to further rising cost. ${ }^{1,7}$

Our study also looked to identify possible patient demographics and relevant risk factors that may predispose to presenting with a UTI as shown in Table I. The mean age of patients presenting was 80.1 years. The majority of patients with a UTI from home were 
female (85\%) with only three male patients also having a positive urine sample. The prevalence specific to sex was $57 \%$ for females and $30 \%$ for males. This compares well with work done previously by Dolk et al. in which they also showed their typical patient profile at highest risk as elderly female patients. ${ }^{15}$

Statistical analysis of all the risk factors found a relative significance with regard to female sex to increase the prevalence of pre-existing UTI ( $p=0.149)$ with an odds ratio of $3: 1$. Age above 80 years seems to be a confounding factor when it is added to female sex, significantly increasing the odds ratio to $5: 1(p=0.068)$. Other risk factors such as fracture type $(p=0.527)$ and history of previous fractures $(p=0.480)$ were found to be less significant as independent risk factors. The ambulatory status of the patient and if they presented from a care facility was not significant. Elderly female patients (over age 80 years) with proximal femur fractures are thus at a five times greater risk of presenting with a UTI from home. Larger studies are, however, needed to evaluate risk factors more accurately.

The most common organism identified was Escherichia coli. This organism was cultured in 11 of the 20 patients with a positive urine culture sample (Figure 1). The majority of the Escherichia coli cultured were sensitive to more commonly used antibiotics like ciprofloxacin, nitrofurantoin and amoxicillin/clavulanic acid. Most of the Escherichia coli showed resistance to amoxicillin/ ampicillin and trimethoprim/sulfamethoxazole (Table II). This is an area of concern as these traditionally acceptable empirical and prophylactic antibiotic choices for management of UTI seem to be becoming more resistant. The results also suggest a possible shift from using ciprofloxacin or nitrofurantoin as first choice of cover for this organism to amoxicillin/clavulanic acid. Organisms cultured other than Escherichia coli showed a tendency to be more drug resistant.

The bacterial organism profile identified in our study correlates with the retrospective analysis done by Yassa et al. in patients with hip fractures where they also found the most common causative organisms of the urinary tract to be Escherichia coli, followed by mixed growth, Enteroccocus faecalis and Pseudomonas aeriginosa. ${ }^{12}$

A retrospective study done by Habte et al. at Dr George Mukhari Hospital in Ga-Rankuwa, Pretoria, looked at the profile of common uropathogens isolated from urine specimens of 2203 samples received; they reported a high urine sample culture positive rate of $51.1 \%$ (1 126 samples) with a greater incidence among females (64\%). ${ }^{16}$ Escherichia coli was the most common aetiological agent identified (39\%) with most organisms still susceptible to nitrofurantoin. Resistance levels to amoxicillin/ampicillin and trimethoprim/sulfamethoxazole were very high and thus they were not recommended for the empiric treatment of UTIs. This study did not look at the use of amoxicillin/clavulanic acid or ciprofloxacin. ${ }^{16}$ The findings of our study are consistent with this study by Habte et al. and show similar results.

Langenhan et al. conducted a retrospective studies study of 1089 geriatric patents with proximal femoral fractures to evaluate the current strategies for peri-operative prophylaxis of infections after operative stabilisation. They concluded that immediate antibiotic therapy of a prevalent bacteriuria for five days decreases the risk of surgical site infection. ${ }^{17}$ They used ciprofloxacin as the first choice for empirical treatment of UTI in conjunction with the standard single dose peri-operative cephalosporin (cefuroxime) at induction of anaesthesia. Ciprofloxacin offers good cover against the organisms like Escherichia coli, Proteus mirabilis, Pseudomonas aeruginosa and the Enterococcus found in UTI as well as those organisms found in surgical site infection in this specific group. This treatment was shown to be effective for this specific group of patients with concomitant hip fracture and UTI. ${ }^{17}$
The data from our study suggests that ciprofloxacin, nitrofurantoin and amoxicillin/clavulanic acid remain good choices for treatment of UTIs in our population. Amoxicillin/clavulanic acid may have a more important role in the future.

Re-evaluation of our current strategies for the use of antibiotics in orthopaedic surgery is needed however, and evidence-based therapy protocols have to be developed by doing larger multicentre

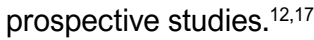

Our study has some limitations. A relatively small number of patients were included in the study. This was attributed to strict inclusion criteria and that the centre where the study was conducted is a tertiary referral hospital. Most patients were transferred to this facility already having had a urinary catheter inserted were excluded. Larger prospective studies will still need to be done at multiple centres. ${ }^{16}$ Another limitation was that there was no follow-up as to whether patients that had a positive urine sample culture were subsequently treated with antibiotics. It was thus not possible to evaluate if this affected the overall prognosis and outcome of the patient or whether this contributed significantly to the development of the discussed complications. Our observations and results only point out the potential complications of UTI in elderly patients and those who are at risk, and does not make any clear treatment recommendation with regard to the indications for treatment, antibiotic classes or duration. These limitations present the opportunity for potential areas of future evaluation.

\section{Conclusion}

The prevalence of UTI in elderly patients with proximal femoral insufficiency fractures is underestimated and higher than expected, with our results suggesting up to $50 \%$ of patients being affected. Higher risk patients for presenting with a UTI from home are the elderly female patients above 80 years of age. Screening for, diagnosing and treating UTIs earlier has clear benefits and potentially decreases overall morbidity and mortality in this specific group of patients. By identifying the most common organisms and sensitivity profile, patients at a higher risk could be treated more effectively and the most common organism targeted more specifically. Protocols and evidence-based therapy strategies have to be developed in larger multicentre prospective studies for these specific high-risk patients.

\section{Ethics statement}

Ethical clearance was obtained from the Department of Research Ethics at the University of Pretoria (reference no. 449/2014) and permission to conduct research was obtained from the management at Steve Biko Academic Hospital before the research started. The study was performed in accordance with the ethical standards of the Helsinki Declaration of 1975 as revised in 2008.

\section{References}

1. Hedström M, Gröndal L, Ahl T. Urinary tract infection in patients with hip fractures. Injury 1999;30(5):341-43.

2. Dolk T. Hip fractures: treatment and early complications. Ups $J$ Med Sci 1989;94(2):195-207.

3. Merchant $\mathrm{R}$, Lui $\mathrm{K}$, Ismail N, Wong $\mathrm{H}$, Sitoh $\mathrm{Y}$. The relationship between postoperative complications and outcomes after hip fracture surgery. Ann Acad Med Singap 2005;34(2):163-68.

4. Brooks J. Using the National Healthcare Safety Network for CAUTI Surveillance. Centers for Disease Control and Prevention (CDC). Division of Healthcare Quality Promotion 2012.

5. Egol KA, Strauss EJ. Perioperative considerations in geriatric patients with hip fracture: what is the evidence? J Orthop Trauma 2009 Jul;23(6):386-94. 
6. Da Chuna PT, Artifon AN, Lima DP, Marques WV, Rahal MA, Ribeiro RR, Kitadai FT. Hip fractures in the elderly: Surgical treatment timing and its correlation with delirium and infection. Acta Orthop Bras 2008;16(3):173-76.

7. Poh KS, Lingaraj K. Complications and their risk factors following hip fracture surgery. Journal of Orthopaedic Surgery 2013;21(2).

8. McGuire KJ, Bernstein J, Polsky D. Delays until surgery after hip fracture increases mortality. Clin Orthop Relat Res 2004;428:294-301.

9. Dolk T, Dolk T. Influence of treatment factors on the outcome after hip fractures. Ups J Med Sci 1989;94(2):209-21.

10. Westberg M, Snorrason F, Frihagen F. Preoperative waiting time increased the risk of periprosthetic infection in patients with femoral neck fracture. Acta orthopaedica 2013;84(2):124-29.

11. Johnstone DJ, Morgan NH, Wilkinson MC, Chissell HR. Urinary tract infection and hip fracture. Injury 1995 3;26(2):89-91.

12. Yassa RR, Khalfaoui MY, Veravalli K, Evans DA; Pre-operative urinary tract infection: is it a risk factor for early surgical site infection with hip fracture surgery? A retrospective analysis. JRSM Open 2017;1;8(3).

13. Carpintero P, Caeiro JR, Carpintero R, Morales A, Silva S, Mesa M. Complications of hip fractures: A review. World $J$ Orthop 2014;5(4): 402-11.

14. Dovjak $\mathrm{P}$, Iglseder $\mathrm{B}$, Mikosch $\mathrm{P}$, Gosch M, Müller E, Pinter G, Pils K, Gerstofer I, Thaler H, Zmaritz M, WeissenbergerLeduc M, Müller W. Treatment and prevention of postoperative complications in hip fracture patients: infections and delirium. Wien Med Wochenschr 2013;163:448-54.

15. Dolk T. Hip fractures-patient background. Ups J Med Sci 1989;94(2):183-94.

16. Habte TM, Dube S, Ismail N, Hoosen AA. Hospital and community isolates of uropathogens at a tertiary hospital in South Africa. $S$ Afr Med J 2009;99:584-87.

17. Langenhan R, Bushuven S, Reimers N, Probst A. Peri-operative antibiotic treatment of bacteriuria reduces early deep surgical site infections in geriatric patients with proximal femur fracture. Int Orthop 2017. 